\title{
Hopf Bifurcation Analysis and Chaos Control of a Chaotic System without Silnikov Orbits
}

\author{
Na Li, ${ }^{1}$ Wei Tan, ${ }^{1,2}$ and Huitao Zhao ${ }^{1}$ \\ ${ }^{1}$ Department of Mathematics and Statistics, Zhoukou Normal University, Zhoukou, Henan 466001, China \\ ${ }^{2}$ Department of Mathematics and Information Science, Beifang University of Nationalities, Yinchuan, Ningxia 750021, China \\ Correspondence should be addressed to Huitao Zhao; taohuiz@sohu.com
}

Received 14 September 2015; Accepted 23 November 2015

Academic Editor: Luca Guerrini

Copyright (c) $2015 \mathrm{Na} \mathrm{Li}$ et al. This is an open access article distributed under the Creative Commons Attribution License, which permits unrestricted use, distribution, and reproduction in any medium, provided the original work is properly cited.

\begin{abstract}
This paper mainly investigates the dynamical behaviors of a chaotic system without Silnikov orbits by the normal form theory. Both the stability of the equilibria and the existence of local Hopf bifurcation are proved in view of analyzing the associated characteristic equation. Meanwhile, the direction and the period of bifurcating periodic solutions are determined. Regarding the delay as a parameter, we discuss the effect of time delay on the dynamics of chaotic system with delayed feedback control. Finally, numerical simulations indicate that chaotic oscillation is converted into a steady state when the delay passes through a certain critical value.
\end{abstract}

\section{Introduction}

In the past decades, both the chaotic synchronization and chaos control have excited amusements in many areas of science and technology due to their potential application. As is known some chaos is desirable and needs not to be controlled, but others are undesirable and need to be controlled. Therefore, the investigation of chaotic control is of great significance. Moreover, many schemes have been presented to implement chaotic control [1-18], of which the time-delayed feedback controlling forces is proved to be a simple and viable method for a continuous dynamical system. Fortunately, the time-delayed feedback controller can also be used to realize the control of a bifurcation. As is well discussed the chaotic dynamical behaviors of the chaotic system will be varied if the steady state is stable or the bifurcating periodic solutions are orbitally asymptotically stable.

Some authors study the chaotic systems with S̃inikov orbit, such as Elhadj and Sprott [9]. Some others investigate chaotic systems without Ŝilnikov orbit, like Wang and Chen [19-21], and so on. Recently, a chaotic system of non-Ŝilnikov type has been introduced in $[19,22]$ as follows:

$$
\begin{aligned}
& \dot{x}=a(y-x), \\
& \dot{y}=-a x-b y z, \\
& \dot{z}=-c+y^{2},
\end{aligned}
$$

where $a>0, b>0$, and $c>0$. The bifurcations and period orbits of (1) will appear taking $b$ as the bifurcation parameter values and fixing $a=40, c=10$. When $b=33$ especially, there appears a chaotic attractor (see Figure 1). The system has two equilibria: $E_{1}(\sqrt{c}, \sqrt{c},-a / b), E_{2}(-\sqrt{c},-\sqrt{c},-a / b)$.

Inspired by the above, in this paper, we focus on the control of chaotic attractor of chaotic system (1). In order to better reflect the dynamical behaviors of system (1) depending on the past system information, it is reasonable to incorporate time delay into this system. The signal error of the current state and the past state of the continuous time system will be fed back to the system itself. Following the idea of Pyragas [13], we add a time-delayed force $k[y(t)-y(t-\tau)]$ to the second equation of system (1) and then system (1) takes the form as follows:

$$
\begin{aligned}
& \dot{x}(t)=a(y(t)-x(t)), \\
& \dot{y}(t)=-a x(t)-b y(t) z(t)+k[y(t)-y(t-\tau)], \\
& \dot{z}(t)=-c+y^{2}(t),
\end{aligned}
$$

where $k$ is a real constant.

The structure of this paper is organized as follows. In Section 2, the stability and the existence of Hopf bifurcation are determined. In Section 3, based on the normal form method and the center manifold theorem presented in 

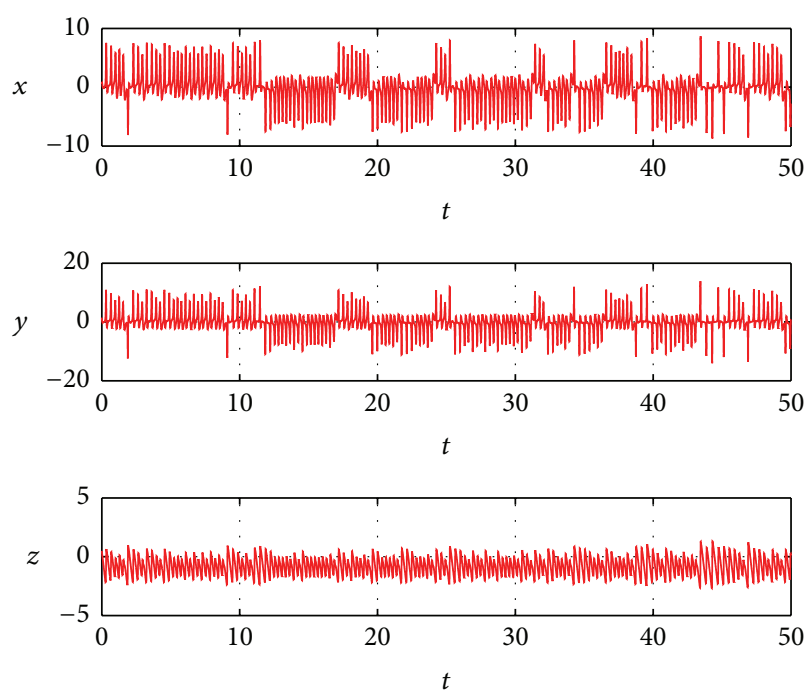

(a) The trajectories

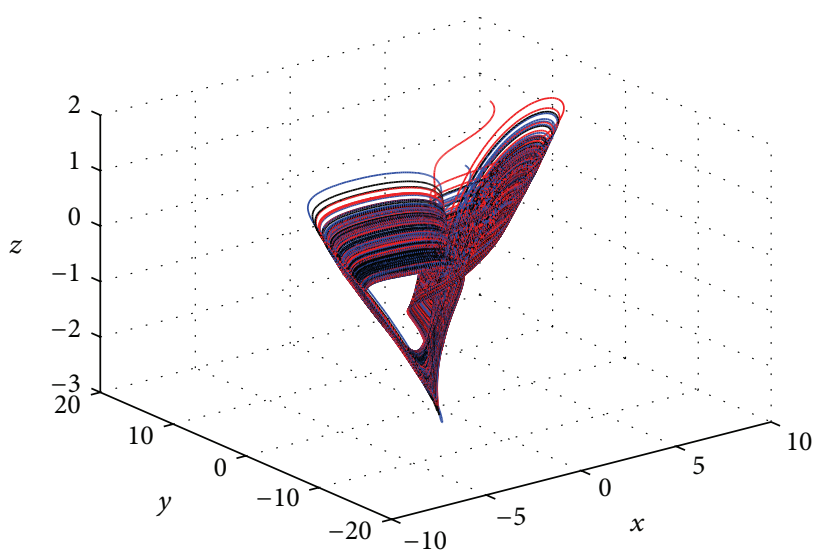

(b) Phase graph

FIGURE 1: The trajectories and phase graphs of system (2) with $\tau=0$.

Hassard et al. [23], the formulae of determining the direction, the stability, and the period of the bifurcating period solutions are presented. To verify the theoretic analysis, numerical simulations are given in Section 4. Finally, a summary of the main conclusions is provided in Section 5.

\section{Bifurcation Analysis of the Chaotic System without Silnikov Orbits}

In this section, we analyze the influence of time delay on the stability of the steady state by taking $\tau$ as bifurcation parameter and fixing the value of $k$. The stability of system (2) will be changed and a family of periodic orbits will bifurcate from the equilibria with the increasing of $\tau$.

By the linear transformation

$$
\begin{aligned}
& x_{1}=x-x_{0}, \\
& y_{1}=y-y_{0}, \\
& z_{1}=z-z_{0},
\end{aligned}
$$

system (2) becomes

$$
\begin{aligned}
\dot{x}_{1}(t)= & a\left(y_{1}(t)-x_{1}(t)\right), \\
\dot{y}_{1}(t)= & -a x_{1}(t)-b z_{0} y_{1}(t)-b y_{0} z_{1}(t)-b y_{1}(t) z_{1}(t) \\
& +k\left[y_{1}(t)-y_{1}(t-\tau)\right], \\
\dot{z}_{1}(t)= & 2 y_{0} y_{1}(t)+y_{1}^{2}(t) .
\end{aligned}
$$

Then, the Jacobian matrix of (4) at any point $(x, y)$ can be written as

$$
J=\left[\begin{array}{ccc}
-a & a & 0 \\
-a & -b z_{0}+k-k e^{-\lambda \tau} & -b y_{0} \\
0 & 2 y_{0} & 0
\end{array}\right]
$$

The characteristic equation of (4) is given by

$$
\lambda^{3}+a_{2} \lambda^{2}+a_{1} \lambda+a_{0}+\left(b_{2} \lambda^{2}+b_{1} \lambda+b_{0}\right) e^{-\lambda \tau}=0,
$$

where $a_{2}=b z_{0}+a-k, a_{1}=2 b y_{0}^{2}+a^{2}+a b z_{0}-a k$, $a_{0}=2 a b y_{0}^{2}, b_{2}=k, b_{1}=k a$, and $b_{0}=0$. Thus, we will study the distribution of the roots of the third-degree exponential polynomial equation of (6), obviously, if $i \omega(\omega>0)$ is a root of (6) and $\omega$ satisfies

$$
\begin{aligned}
& -i \omega^{3}-a_{2} \omega^{2}+i a_{1} \omega+a_{0} \\
& \quad+\left(i b_{1} \omega-b_{2} \omega^{2}\right)(\cos (\omega t)-i \sin (\omega t))=0 .
\end{aligned}
$$

Separating the real and imaginary parts of (7), we get

$$
\begin{aligned}
& a_{2} \omega^{2}-a_{0}=-b_{2} \omega^{2} \cos (\omega \tau)+b_{1} \omega \sin (\omega \tau), \\
& \omega^{3}-a_{1} \omega=b_{1} \omega \cos (\omega \tau)+b_{2} \omega^{2} \sin (\omega \tau),
\end{aligned}
$$

which is equivalent to

$$
\begin{aligned}
\omega^{6} & +\left(a_{2}^{2}-b_{2}^{2}-2 a_{1}\right) \omega^{4}+\left(a_{1}^{2}-2 a_{0} a_{2}-b_{1}^{2}\right) \omega^{2}+a_{0}^{2} \\
& =0
\end{aligned}
$$

Let $z=\omega^{2}$ and denote $p=a_{2}^{2}-b_{2}^{2}-2 a_{1}, q=a_{1}^{2}-2 a_{0} a_{2}-b_{1}^{2}$, and $r=a_{0}^{2}$; (10) becomes

$$
z^{3}+p z^{2}+q z+r=0
$$

From (11), we have

$$
h(z)=z^{3}+p z^{2}+q z+r,
$$

where $r>0$.

$$
\text { Then, } h^{\prime}(z)=3 z^{2}+2 p z+q \text {. }
$$


Denote $\Delta=p^{2}-3 q \leqslant 0$ and $\Delta>0$; the equation $3 z^{2}+$ $2 p z+q=0$ has two real roots as follows:

$$
\begin{aligned}
& z_{1}^{*}=\frac{-p+\sqrt{\Delta}}{3}, \\
& z_{2}^{*}=\frac{-p-\sqrt{\Delta}}{3} .
\end{aligned}
$$

Notice that $\lim _{z \rightarrow \infty} h(z)=+\infty$ and $r=a_{0}^{2}>0$. We introduce the following results proved by Lü and Chen [24].

Lemma 1. For the polynomial equation (11), we have the following results:

(1) If $\Delta=p^{2}-3 q \leqslant 0$, then (11) does not have positive real root.

(2) If $\Delta=p^{2}-3 q>0$, then (11) has positive real roots if and only if $z_{1}^{*}=(-p+\sqrt{\Delta}) / 3>0$ and $h\left(z_{1}^{*}\right) \leqslant 0$. tion:

Without loss of generality, we give the following assump-

$$
\begin{aligned}
\Delta & =p^{2}-3 q>0, \\
z_{1}^{*} & =\frac{-p+\sqrt{\Delta}}{3} \\
h\left(z_{1}^{*}\right) & \leqslant 0 .
\end{aligned}
$$

If we make an assumption that (14) is true, then (11) has two positive roots $z_{1}$ and $z_{2}$. Supposing $z_{1}<z_{2}$, we have $h^{\prime}\left(z_{1}^{*}\right) \leqslant$ 0 . Substituting $\omega_{k}=\sqrt{z_{k}}(k=1,2)$ into $(8)$, we find that

$$
\begin{aligned}
\tau_{k}^{j} & =\frac{1}{\omega_{k}}\left\{\cos ^{-1}\left(\frac{\left(b_{1}-a_{2} b_{2}\right) \omega_{k}^{2}-\left(a_{1} b_{1}-a_{0} b_{2}\right)}{b_{2}^{2} \omega_{k}^{2}+b_{1}^{2}}\right)\right. \\
& +2 j \pi\},
\end{aligned}
$$

where $(k=1,2$ and $j=0,1,2, \ldots)$; then $\pm i \omega_{k}$ is a pair of pure imaginary roots of (7) with $\tau_{k}^{j}$. Define

$$
\tau_{0}=\tau_{k_{0}}^{0}=\min _{k \in\{1,2,3\}}\left\{\tau_{k}^{0}\right\} .
$$

Lemma 2. If (14) holds, when $\tau=\tau_{k}^{j}(k=1,2 ; j=$ $0,1,2, \ldots)$, then (11) has a pair of pure imaginary roots $\omega_{k}$ and all the other roots of (11) have nonzero real parts.

Lemma 3. If (14) holds, then we have the following transversality condition:

(i) $[d(\operatorname{Re} \lambda) / d \tau]_{\tau=\tau_{k}}^{-1}<0$ and $[d(\operatorname{Re} \lambda) / d \tau]_{\tau=\tau_{k}}^{-1}>0$.

(ii) $[d(\operatorname{Re} \lambda) / d \tau]_{\tau=\tau_{k}^{j}}^{-1}(j=0,1,2, \ldots)$ and $h^{\prime}\left(z_{k}\right)$ have the same sign.

Proof. Substituting $\lambda(\tau)$ into (6) and taking the derivative with respect to $\tau$, we obtain

$$
\left(\frac{d \lambda}{d \tau}\right)^{-1}=\frac{\left(3 \lambda^{2}+2 a_{2} \lambda+a_{1}\right)}{\lambda\left(b_{2} \lambda^{2}+b_{1} \lambda\right) e^{-\lambda \tau}}+\frac{2 b_{2} \lambda+b_{1}}{\lambda\left(b_{2} \lambda^{2}+b_{1} \lambda\right)}-\frac{\tau}{\lambda} .
$$

From (9), we find that

$$
\begin{aligned}
& \left(\frac{d \lambda}{d \tau}\right)_{\tau=\tau_{k}}^{-1}=\operatorname{Re}\left[\frac{\left(3 \lambda^{2}+2 a_{2} \lambda+a_{1}\right)}{\lambda\left(b_{2} \lambda^{2}+b_{1} \lambda\right) e^{-\lambda \tau}}\right]_{\tau=\tau_{k}} \\
& +\operatorname{Re}\left[\frac{2 b_{2} \lambda+b_{1}}{\lambda\left(b_{2} \lambda^{2}+b_{1} \lambda\right)}\right]_{\tau=\tau_{k}} \\
& =\operatorname{Re}\left[\frac { ( 3 \lambda ^ { 2 } + 2 a _ { 2 } \lambda + a _ { 1 } ) } { \lambda ( b _ { 2 } \lambda ^ { 2 } + b _ { 1 } \lambda ) } \left(\cos \left(\omega_{k} \tau_{k}^{j}\right)\right.\right. \\
& \left.\left.+i \sin \left(\omega_{k} \tau_{k}^{j}\right)\right)\right]_{\tau=\tau_{k}}+\operatorname{Re}\left[\frac{2 b_{2} \lambda+b_{1}}{\lambda\left(b_{2} \lambda^{2}+b_{1} \lambda\right)}\right]_{\tau=\tau_{k}} \\
& =\frac{1}{\Lambda}\left[3 \omega_{k}^{6}+2\left(a_{2}^{2}-b_{2}^{2}-2 a_{1}\right) \omega_{k}^{4}+\left(a_{1}^{2}-2 a_{0} a_{2}\right.\right. \\
& \left.\left.-b_{1}^{2}\right) \omega_{k}^{2}\right]=\frac{1}{\Lambda}\left[3 \omega_{k}^{6}+2 p \omega_{k}^{4}+q \omega_{k}^{2}\right]=\frac{z_{k}}{\Lambda}\left[3 z_{k}^{2}\right. \\
& \left.+2 p z_{k}+q\right]=\frac{z_{k}}{\Lambda}\left[h^{\prime}\left(z_{k}\right)\right],
\end{aligned}
$$

where $\Lambda=b_{1}^{2} \omega_{k}^{4}+b_{2}^{2} \omega_{k}^{6}$. Since $z_{k}>0$, we conclude that $(d \lambda / d \tau)_{\tau=\tau_{k}^{j}}$ and $h^{\prime}\left(z_{k}\right)$ have the same sign. This completes the proof.

Theorem 4. Suppose (14) and Routh-Hurwitz criteria for (7) are satisfied. Moreover, if $\tau_{1}^{0}>\tau_{2}^{0}$, there exists $m \in N$ such that $\tau_{2}^{0}<\tau_{1}^{0}<\tau_{2}^{1}<\tau_{1}^{1}<\tau_{2}^{2}<\tau_{1}^{2}<\cdots<\tau_{2}^{m}<\tau_{1}^{m}<\tau_{2}^{m+1}<\tau_{1}^{m+1}$.

(i) For $\tau \in\left[0, \tau_{2}^{0}\right] \cup\left[\tau_{1}^{0}, \tau_{2}^{1}\right] \cup \cdots \cup\left[\tau_{1}^{m-1}, \tau_{2}^{m}\right] \cup\left[\tau_{1}^{m}, \tau_{2}^{m+1}\right]$, the equilibriums $E_{1,2}$ of system (2) are unstable.

(ii) For $\tau \in\left[\tau_{2}^{0}, \tau_{1}^{0}\right] \cup\left[\tau_{2}^{0}, \tau_{1}^{1}\right] \cup \cdots \cup\left[\tau_{2}^{m}, \tau_{1}^{m}\right] \cup\left[\tau_{2}^{m+1},+\infty\right]$ the equilibriums $E_{1,2}$ are asymptotically stable.

(iii) When $\tau=\tau_{k}^{j}(k=1,2$ and $j=0,1,2, \ldots)$, system (2) undergoes a Hopf bifurcation at the equilibriums $E_{1,2}$.

\section{Direction and Stability of Hopf Bifurcation Period Solution}

In the previous section, we obtained the conditions of Hopf bifurcation occurring. In this section, the direction and the stability of the bifurcations are analyzed using the central manifold theorem. Assuming that system (2) always undergoes Hopf bifurcation at the equilibrium $E_{1}$ for $\tau=$ $\tau_{k}^{j}(k=1,2$ and $j=0,1,2, \ldots)$, then, letting $u_{1}=x-x_{0}$, $u_{2}=y-y_{0}, u_{3}=z-z_{0}, \bar{u}_{i}=u_{i}(t \tau)$, and $\tau=\tau_{k}^{j}$ and dropping the bars for simplifications of notations, the nonlinear system (2) can be transformed into an $\mathrm{FDE}$ in $C \in C\left([-1,0], R^{3}\right)$ as

$$
\dot{u}(t)=L_{\mu}\left(u_{t}\right)+f\left(\mu, u_{t}\right),
$$


where $u(t)=\left(u_{1}(t), u_{2}(t), u_{3}(t)\right)^{T} \in R^{3}$ and $L_{\mu}: C \rightarrow R^{3}$ and $f: R \times C \rightarrow R$ are given, respectively, by

$$
\begin{aligned}
L_{\mu}(\varphi)= & \left(\mu+\tau_{k}\right)\left(\begin{array}{ccc}
-a & a & 0 \\
-a & k-b z_{0} & -b y_{0} \\
0 & 2 y_{0} & 0
\end{array}\right)\left(\begin{array}{l}
\varphi_{1}(0) \\
\varphi_{2}(0) \\
\varphi_{3}(0)
\end{array}\right) \\
& +\left(\mu+\tau_{k}\right)\left(\begin{array}{ccc}
0 & 0 & 0 \\
0 & -k & 0 \\
0 & 0 & 0
\end{array}\right)\left(\begin{array}{c}
\varphi_{1}(-1) \\
\varphi_{2}(-1) \\
\varphi_{3}(-1)
\end{array}\right), \\
f(\tau, \varphi)= & \left(\mu+\tau_{k}\right)\left(\begin{array}{c}
0 \\
-b \varphi_{2}(0) \varphi_{3}(0) \\
\varphi_{2}^{2}(0)
\end{array}\right) .
\end{aligned}
$$

If we choose

$$
\begin{aligned}
\eta(\theta, \mu)= & \left(\mu+\tau_{k}\right)\left(\begin{array}{ccc}
-a & a & 0 \\
-a & k-b z_{0} & -b y_{0} \\
0 & 2 y_{0} & 0
\end{array}\right) \delta(\theta) \\
& -\left(\mu+\tau_{k}\right)\left(\begin{array}{ccc}
0 & 0 & 0 \\
0 & -k & 0 \\
0 & 0 & 0
\end{array}\right) \delta(\theta+1),
\end{aligned}
$$

where $\delta(\cdot)$ is a Dirac delta function and $\theta \in[-1,0]$, then there is

$$
L_{\mu}(\varphi)=\int_{-1}^{0} d \eta(\theta, \mu) \varphi(\theta), \quad \text { for } \theta \in C .
$$

For $\varphi \in C\left([-1,0], R^{3}\right)$, define

$$
\begin{aligned}
& A(\mu) \varphi= \begin{cases}d \frac{\varphi(\theta)}{d \theta}, & \theta \in(0,1], \\
\int_{-1}^{0} d \eta(\theta, s) \varphi(s), & \theta=0,\end{cases} \\
& R(\mu) \varphi= \begin{cases}0, & \theta \in(0,1], \\
F(\mu, \varphi), & \theta=0 .\end{cases}
\end{aligned}
$$

For convenience, we can write system (9) into an operator equation

$$
\dot{u}(t)=A(\mu) u_{t}+R(\mu) u_{t},
$$

where $u_{t}(\theta)=u(t+\theta), \theta \in[-1,0]$.

For $\psi \in\left([-1,0],\left(R^{3}\right)^{*}\right)$, define

$$
A^{*} \varphi(s)= \begin{cases}-\frac{d \psi(s)}{d \theta}, & s \in(0,1], \\ \int_{-1}^{0} d \eta^{T}(t, 0) \psi(-t), & s=0,\end{cases}
$$

and a bilinear inner product

$$
\begin{aligned}
\langle\psi, \varphi\rangle= & \overline{\psi(0)} \varphi(0) \\
& -\int_{\theta=-1}^{0} \int_{\xi=0}^{\theta} \bar{\psi}^{T}(\xi-\theta) d \eta(\theta) \varphi(\xi) d \xi,
\end{aligned}
$$

where $\eta(\theta)=\eta(\theta, 0)$. Obviously, $A^{*}$ and $A(0)$ are adjoint operators. In line with the discussion in Section 2, we know that $\pm i \omega_{k} \tau_{k}$ are eigenvalues of $A(0)$. Thus they are eigenvalues of $A^{*}$. Next, we need to calculate the eigenvectors of $A^{*}$ and $A(0)$ corresponding to $-i \omega_{k} \tau_{k}$ and $i \omega_{k} \tau_{k}$, respectively. Let $q(\theta)=(1, \alpha, \beta)^{T} e^{i \theta \omega \tau}$ be the eigenvalue of $A(0)$; that is, $A(0) q(0)=A(\theta)=i \omega_{k} q(\theta)$; we have

$$
\begin{aligned}
& \tau\left(\begin{array}{ccc}
i \omega_{k}+a & -a & 0 \\
a & i \omega_{k}-k+b z_{0}+k e^{-i \omega_{k} \tau} & b y_{0} \\
0 & -2 y_{0} & i \omega_{k}
\end{array}\right) q(0) \\
& =\left(\begin{array}{l}
0 \\
0 \\
0
\end{array}\right) .
\end{aligned}
$$

Then it is easy to obtain

$$
q(0)=(1, \alpha, \beta)^{T}=\left(1, \frac{i \omega_{k}+a}{a}, \frac{2 y_{0}\left(i \omega_{k}+a\right)}{i \omega_{k} a}\right)^{T} .
$$

Similarly, supposing $q^{*}(\theta)=\left(1, \alpha^{*}, \beta^{*}\right)^{T} e^{i s \omega \tau}$, from the definition of $A^{*}$, we have

$$
\begin{aligned}
q^{*}(0) & =D\left(1, \alpha^{*}, \beta^{*}\right)^{T} \\
& =D\left(1, \frac{a-i \omega_{k}}{a}, \frac{b y_{0}\left(a-i \omega_{k}\right)}{i \omega_{k} a}\right)^{T} .
\end{aligned}
$$

So we have

$$
\begin{aligned}
q(\theta) & =(1, \alpha, \beta)^{T} \\
& =\left(1, \frac{i \omega_{k}+a}{a}, \frac{2 y_{0}\left(i \omega_{k}+a\right)}{i \omega_{k} a}\right)^{T} e^{i \omega_{0} \tau_{k} \theta}, \\
& \theta \in[-1,0], \\
q^{*}(s) & =D\left(1, \alpha^{*}, \beta^{*}\right)^{T} \\
& =D\left(1, \frac{a-i \omega_{k}}{a}, \frac{b y_{0}\left(a-i \omega_{k}\right)}{i \omega_{k} a}\right)^{T} e^{i \omega_{0} \tau_{k} s}, \\
s & \in[0,1],
\end{aligned}
$$

where $D$ is a constant such that $\left\langle q^{*}(s), q(\theta)\right\rangle=1$. By (26), we get

$$
\begin{aligned}
& \left\langle q^{*}(s), q(\theta)\right\rangle=\bar{D}\left(1, \overline{\alpha^{*}}, \overline{\beta^{*}}\right)(1, \alpha, \beta)^{T} \\
& \quad-\int_{-1}^{0} \int_{\xi=0}^{\theta} \bar{D}\left(1, \overline{\alpha^{*}}, \overline{\beta^{*}}\right) e^{-i(\xi-\theta) \omega \tau} d \eta(\theta)(1, \alpha, \beta)^{T} \\
& \cdot e^{i \xi \omega \tau} d \xi=\bar{D}\left(1+\overline{\alpha^{*}} \alpha+\overline{\beta^{*}} \beta\right) \\
& \quad-\int_{-1}^{0} \int_{\xi=0}^{\theta} \bar{D}\left(1, \overline{\alpha^{*}}, \overline{\beta^{*}}\right) \theta e^{i \theta \omega \tau} d \eta(\theta)(1, \alpha, \beta)^{T} \\
& =\bar{D}\left(1+\overline{\alpha^{*}} \alpha+\overline{\beta^{*}} \beta+k \tau_{k} \overline{\alpha^{*}} \alpha e^{-i \omega_{k} \tau_{k}}\right)=1 .
\end{aligned}
$$


Therefore, we can choose $D$ as

$$
D=\frac{1}{\left(1+\overline{\alpha^{*}} \alpha+\overline{\beta^{*}} \beta+k \tau_{k} \overline{\alpha^{*}} \alpha e^{-i \omega_{k} \tau_{k}}\right)} .
$$

Below we apply the idea in Hassard et al. [23] to compute the coordinates describing the center manifold coat $\mu=0$. Let $x_{t}$ be the solution of (2) when $x=0$. Define

$$
\begin{aligned}
z(t) & =\left\langle q^{*}, x_{t}\right\rangle, \\
W(t, \theta) & =x_{t}(\theta)-2 \operatorname{Re} z(t) q(\theta) .
\end{aligned}
$$

On the center manifold $C_{0}$, we get

$$
W(t, \theta)=W(z(t), \overline{z(t)}, \theta)
$$

where

$$
\begin{aligned}
W(t, \theta) & =W(z(t), \overline{z(t)}, \theta)=W(z, \bar{z}) \\
& =W_{20} \frac{z^{2}}{2}+W_{11} z \bar{z}+W_{02} \frac{\bar{z}^{2}}{2}+\cdots
\end{aligned}
$$

and $z$ and $\bar{z}$ are local coordinates for center manifold $C_{0}$ in the direction of $q^{*}$ and $\overline{z^{*}}$. Noting that $W$ is also real if $x_{t} \in C_{0}$ of (2), so we deal with real solutions only. For solution $x_{t}$, since $x=0$, we have

$$
\begin{aligned}
\dot{z}= & \left\langle q^{*}(s), \dot{x}(t)\right\rangle=\left\langle q^{*}(s), A(0) x_{t}+R(0) x_{t}\right\rangle \\
= & \left\langle q^{*}(s), A(0) x_{t}\right\rangle+\left\langle q^{*}(s), R(0) x_{t}\right\rangle \\
& \quad-\int_{-1}^{0} \int_{\xi=0}^{\theta} \overline{q^{*}}(\xi-\theta) \eta(\theta) A(0) R(0) x_{t}(\xi) d(\xi) \\
= & \left\langle i \omega_{0} \tau_{k} q^{*}(s), x_{t}\right\rangle+\overline{q^{*}}(0) f\left(0, x_{t}(\theta)\right) \\
= & i \omega_{0} \tau_{k} z(t)+\overline{q^{*}}(0) f_{0}(z(t), \overline{z(t)}) .
\end{aligned}
$$

That is,

$$
\dot{z}(t)=i \omega_{0} \tau_{k} z+g(z, \bar{z})
$$

where

$$
g(z, \bar{z})=g_{20} \frac{z^{2}}{2}+g_{11} z \bar{z}+g_{02} \frac{\bar{z}^{2}}{2}+\cdots
$$

Then we have

$$
\begin{aligned}
& g(z, \bar{z})=\overline{q^{*}(0)} f_{0}(z, \bar{z})=f\left(0, x_{t}\right) \\
& \quad=\bar{D} \tau_{k}\left(1, \alpha^{*}, \beta^{*}\right)\left(f_{1}\left(0, x_{t}\right), f_{2}\left(0, x_{t}\right), f_{3}\left(0, x_{t}\right)\right)^{T},
\end{aligned}
$$

where

$$
\begin{aligned}
& f_{1}\left(0, x_{t}\right)=0, \\
& f_{2}\left(0, x_{t}\right)=-\tau_{k} b y_{1 t}(0) z_{1 t}(0), \\
& f_{3}\left(0, x_{t}\right)=\tau_{k} y_{1 t}^{2}(0) .
\end{aligned}
$$

Noticing $x_{t}(\theta)=\left(x_{1 t}(\theta), y_{1 t}(\theta), z_{1 t}(\theta)\right)^{T}=W(t, 0)+z q(\theta)+$ $\bar{z} \bar{q}(\theta)$ and $q(\theta)=(1, \alpha, \beta)^{T} e^{i \omega_{0} \theta}$, we have

$$
\begin{aligned}
x_{1 t}(0)= & z+\bar{z}+W_{20}^{(1)}(0) \frac{z^{2}}{2}+W_{11}^{(1)}(0) z \bar{z} \\
& +W_{02}^{(1)}(0) \frac{\bar{z}^{2}}{2}+\cdots, \\
y_{1 t}(0)= & \alpha z+\bar{\alpha} \bar{z}+W_{20}^{(2)}(0) \frac{z^{2}}{2}+W_{11}^{(2)}(0) z \bar{z} \\
& +W_{02}^{(2)}(0) \frac{\bar{z}^{2}}{2}+\cdots, \\
z_{1 t}(0)= & \beta z+\bar{\beta} \bar{z}+W_{20}^{(3)}(0) \frac{z^{2}}{2}+W_{11}^{(3)}(0) z \bar{z}
\end{aligned}
$$

$$
+W_{02}^{(3)}(0) \frac{\bar{z}^{2}}{2}+\cdots .
$$

In view of (38), (39), and (41), we get

$$
\begin{aligned}
& g(z, \bar{z})=\overline{q^{*}(0)} f_{0}(z, \bar{z})=f\left(0, x_{t}\right) \\
& \quad=\bar{D} \tau_{k}\left(1, \alpha^{*}, \beta^{*}\right)\left(f_{1}\left(0, x_{t}\right), f_{2}\left(0, x_{t}\right), f_{3}\left(0, x_{t}\right)\right)^{T} \\
& \quad=\bar{D} \tau_{k}\left[M z^{2}+N z \bar{z}+P \bar{z}^{2}+Q z^{2} \bar{z}+\cdots\right],
\end{aligned}
$$

where

$$
\begin{aligned}
M & =b \overline{\alpha^{*}} \alpha \beta+\alpha^{2} \overline{\beta^{*}}, \\
N & =b \overline{\alpha^{*}} \alpha \beta+2 \alpha \bar{\alpha} \overline{\beta^{*}}, \\
P & =b \bar{\alpha} \bar{\beta} \overline{\alpha^{*}}+\overline{\beta^{*}} \bar{\alpha}^{2}, \\
Q & =b \overline{\alpha^{*}}\left[\alpha W_{11}^{(3)}(0)+\frac{1}{2} \bar{\alpha} W_{20}^{(3)}(0)+\frac{1}{2} \bar{\beta} W_{20}^{(2)}(0)\right. \\
& \left.+\beta W_{11}^{(2)}(0)\right]+\overline{\beta^{*}}\left[\bar{\alpha} W_{20}^{(2)}(0)+2 \alpha W_{11}^{(2)}(0)\right],
\end{aligned}
$$

where

$$
\begin{aligned}
& g_{20}=2 \bar{D} \tau_{k} M, \\
& g_{11}=\bar{D} \tau_{k} N, \\
& g_{02}=2 \bar{D} \tau_{k} P, \\
& g_{21}=2 \bar{D} \tau_{k} Q .
\end{aligned}
$$

Since $W_{11}^{(3)}(0), W_{20}^{(3)}(0), W_{20}^{(2)}(0)$, and $W_{11}^{(2)}(0)$ in $g_{21}$ are unknown, we still need to compute them. By (24) and (36), we drive

$\dot{W}$

$$
= \begin{cases}A W-2 \operatorname{Re}\left\{\overline{q^{*}(0)} f_{0} q(\theta)\right\}, & \theta \in[-1,0), \\ A W-2 \operatorname{Re}\left\{\overline{q^{*}(0)} f_{0} q(\theta)\right\}+f_{0}, & \theta=0 .\end{cases}
$$


We can rewrite

$$
\dot{W}=A W+H(z, \bar{z}, \theta)
$$

where

$$
\begin{aligned}
H(z, \bar{z}, \theta)= & H_{20} \frac{z^{2}}{2}+H_{11} z \bar{z}+H_{02} \frac{\bar{z}^{2}}{2}+H_{21} \frac{z^{2} \bar{z}}{2} \\
& +\cdots
\end{aligned}
$$

Comparing the coefficients, we get

$$
\begin{aligned}
\left(A-2 i \omega_{0}\right) W_{20}(\theta 0) & =-H_{20}, \\
A W_{11}(\theta) & =-H_{11}(\theta) .
\end{aligned}
$$

For $\theta \in[-1,0)$,

$$
\begin{aligned}
H(z, \bar{z}, \theta) & =-\overline{q^{*}}(0) f_{0} q(\theta)-q^{*}(0) \bar{f}_{0} \overline{q(\theta)} \\
& =-g(z, \bar{z}) q(\theta)-\bar{g}(z, \bar{z}) \bar{q}(\theta) .
\end{aligned}
$$

Comparing the coefficients of (49) with (50), we find that

$$
\begin{aligned}
& H_{20}(\theta)=-g_{20} q(\theta)-\bar{g}_{02} \bar{q}(\theta), \\
& H_{11}(\theta)=-g_{11} q(\theta)-\bar{g}_{11} \bar{q}(\theta) .
\end{aligned}
$$

By (48) and (51) and the definition of $A$, we obtain

$$
\dot{W}_{20}=2 i \omega_{0} \tau_{k} W_{20}(\theta)+g_{20} q(\theta)+\bar{g}_{02} \bar{q}(\theta) .
$$

Since $q(\theta)=q(0) e^{i \omega_{0} \tau_{k} \theta}$, we get

$$
\begin{aligned}
W_{20}(\theta)= & \frac{i g_{20}}{\omega_{0} \tau_{k}} q(0) e^{i \omega_{0} \tau_{k} \theta}+\frac{i \bar{g}_{02}}{3 \omega_{0} \tau_{k}} \bar{q}(0) e^{-i \omega_{0} \tau_{k} \theta} \\
& +E_{1} e^{2 i \omega_{0} \tau_{k} \theta}
\end{aligned}
$$

where $E_{1}=\left(E_{1}^{(1)}, E_{1}^{(2)}, E_{1}^{(3)}\right)^{T}$ is a constant vector.

Similarly, in view of (49) and (52) and the definition of $A$, we get

$$
\begin{aligned}
\dot{W}_{11}(\theta)= & g_{11} q(\theta)+\bar{g}_{11} \bar{q}(\theta) \\
W_{11}(\theta)= & -\frac{i g_{11}}{\omega_{0} \tau_{k}} q(0) e^{i \omega_{0} \tau_{k} \theta}+\frac{i \bar{g}_{11}}{\omega_{0} \tau_{k}} \bar{q}(0) e^{-i \omega_{0} \tau_{k} \theta} \\
& +E_{2},
\end{aligned}
$$

where $E_{1}=\left(E_{2}^{(1)}, E_{2}^{(2)}, E_{2}^{(3)}\right)^{T}$ is a constant vector.

Next, we will seek appropriate $E_{1}, E_{2}$ in (54) and (55), respectively. By means of the definition of $A,(51)$ and (52), we have

$$
\begin{aligned}
& \dot{W}_{20}(\theta)=\int_{-1}^{0} d \eta(\theta) W_{20}(\theta)=2 i \omega_{0} \tau_{k} W_{20}-H_{20}(0) \\
& \dot{W}_{11}(\theta)=\int_{-1}^{0} d \eta(\theta) W_{11}(\theta)=-H_{11}(0)
\end{aligned}
$$

where $\eta(\theta)=\eta(0, \theta)$. In view of (48), we get

$$
\begin{aligned}
& H_{20}(\theta)=-g_{20} q(\theta)-\bar{g}_{02} \bar{q}(\theta)+2 \tau_{k}\left(0, b \alpha \beta, \alpha^{2}\right)^{T}, \\
& H_{11}(\theta)=-g_{11} q(\theta)-\bar{g}_{11} \bar{q}(\theta)+2 \tau_{k}(0, b \alpha \beta, 2 \alpha \bar{\alpha})^{T} .
\end{aligned}
$$

For $i \omega_{k} \tau_{k}$ is the eigenvalues of $A(0)$ and $q(0)$ is the corresponding eigenvector, we obtain

$$
\begin{aligned}
& \left(i \omega_{k} \tau_{k}-\int_{-1}^{0} e^{i \theta \omega \tau} d \eta(\theta)\right) q(0)=0, \\
& \left(-i \omega_{k} \tau_{k}-\int_{-1}^{0} e^{-i \theta \omega \tau} d \eta(\theta)\right) E_{1}=2 \tau_{k}\left(0, b \alpha \beta, \alpha^{2}\right)^{T} .
\end{aligned}
$$

Substituting (54) and (57) into (59), we obtain

$$
\left(2 i \omega_{k} \tau_{k}-\int_{-1}^{0} e^{2 i \theta \omega \tau} d \eta(\theta)\right) E_{1}=2 \tau_{k}\left(0, b \alpha \beta, \alpha^{2}\right)^{T} .
$$

That is,

$$
\begin{aligned}
& \left(\begin{array}{ccc}
2 i \omega_{k}+a & -a & 0 \\
a & 2 i \omega_{k}-k+b z_{0}+k e^{-i \omega_{k} \tau} & b y_{0} \\
0 & -2 y_{0} & 2 i \omega_{k}
\end{array}\right) E_{1} \\
& =\left(\begin{array}{c}
0 \\
b \alpha \beta \\
\alpha^{2}
\end{array}\right) .
\end{aligned}
$$

It follows that

$$
\begin{aligned}
& E_{1}^{(1)}=\frac{\Delta_{11}}{\Delta_{1}}, \\
& E_{1}^{(2)}=\frac{\Delta_{12}}{\Delta_{1}}, \\
& E_{1}^{(3)}=\frac{\Delta_{13}}{\Delta_{1}},
\end{aligned}
$$

where

$$
\begin{aligned}
& \Delta_{11}=2\left|\begin{array}{ccc}
0 & -a & 0 \\
b \alpha \beta & 2 i \omega_{k}-k+b z_{0}+k e^{-i \omega_{k} \tau} & b y_{0} \\
\alpha^{2} & -2 y_{0} & 2 i \omega_{k}
\end{array}\right|, \\
& \Delta_{12}=2\left|\begin{array}{ccc}
2 i \omega_{k}+a & 0 & 0 \\
a & b \alpha \beta & b y_{0} \\
0 & \alpha^{2} & 2 i \omega_{k}
\end{array}\right| \\
& \Delta_{13}=2\left|\begin{array}{ccc}
2 i \omega_{k}+a & -a & 0 \\
a & 2 i \omega_{k}-k+b z_{0}+k e^{-i \omega_{k} \tau} & b \alpha \beta \\
0 & -2 y_{0} & \alpha^{2}
\end{array}\right| \text {, } \\
& \Delta_{1}=\left|\begin{array}{ccc}
2 i \omega_{k}+a & -a & 0 \\
a & 2 i \omega_{k}-k+b z_{0}+k e^{-i \omega_{k} \tau} & b y_{0} \\
0 & -2 y_{0} & 2 i \omega_{k}
\end{array}\right| \text {. }
\end{aligned}
$$


Similarly, substituting (51), (52), and (60) into (58), we have

$$
\begin{aligned}
& \left(\begin{array}{ccc}
a & -a & 0 \\
a & b z_{0} & b y_{0} \\
0 & -2 y_{0} & 0
\end{array}\right) E_{2}=2\left(\begin{array}{c}
0 \\
b \alpha \beta \\
2 \alpha \bar{\alpha}
\end{array}\right), \\
& E_{2}^{(1)}=\frac{\Delta_{21}}{\Delta_{2}}, \\
& E_{2}^{(2)}=\frac{\Delta_{22}}{\Delta_{2}} \text {, } \\
& E_{2}^{(3)}=\frac{\Delta_{23}}{\Delta_{2}}, \\
& \Delta_{21}=2\left|\begin{array}{ccc}
0 & -a & 0 \\
b \alpha \beta & b z_{0} & b y_{0} \\
2 \alpha \bar{\alpha} & -2 y_{0} & 0
\end{array}\right| \text {, } \\
& \Delta_{22}=2\left|\begin{array}{ccc}
a & 0 & 0 \\
a & b \alpha \beta & b y_{0} \\
0 & 2 \alpha \bar{\alpha} & 0
\end{array}\right| \\
& \Delta_{23}=2\left|\begin{array}{ccc}
a & -a & 0 \\
a & b z_{0} & b \alpha \beta \\
0 & -2 y_{0} & 2 \alpha \bar{\alpha}
\end{array}\right|, \\
& \Delta_{2}=\left|\begin{array}{ccc}
a & -a & 0 \\
a & b z_{0} & b y_{0} \\
0 & -2 y_{0} & 0
\end{array}\right| \text {. }
\end{aligned}
$$

Consequently, we can determine $W_{20}(0)$ and $W_{11}(0)$. Thus, all $g_{i j}$ can be determined by [13]. Following the basic idea of [25] and the method in [26], one can draw the conclusion about the bifurcation direction and the stability of the Hopf bifurcation, which are determined by the following parameters:

$$
\begin{aligned}
C_{1}(0) & =\frac{i}{2 \omega_{k} \tau_{k}}\left(g_{20} g_{11}-2\left|g_{11}\right|^{2}-\frac{1}{3}\left|g_{02}\right|^{2}\right)+\frac{g_{21}}{2} \\
\mu_{2} & =-\frac{\operatorname{Re}\left\{C_{1}(0)\right\}}{\operatorname{Re}\{d \lambda / d \tau\}} \\
T_{2} & =\frac{\operatorname{Im}\left\{C_{1}(0)\right\}+\mu_{2} \operatorname{Im}\{d \lambda / d \tau\}}{\omega_{k} \tau_{k}} \\
\beta_{2} & =2 \operatorname{Re}\left\{C_{1}(0)\right\}
\end{aligned}
$$

Thus, we have the main results of this section.

Theorem 5. $\mu_{2}$ determines the direction of the Hopf bifurcation; that is, if $\mu_{2}>0\left(\mu_{2}<0\right)$, the Hopf bifurcation is supercritical (subcritical) and the bifurcating period solutions exist for $\tau>\tau_{k}\left(\tau<\tau_{k}\right) ; \beta_{2}$ determines the stability of the bifurcating period solutions: the bifurcation period solutions are orbitally stable (unstable) if $\beta_{2}<0\left(\beta_{2}>0\right)$, and $T_{2}$ determines the period of the bifurcating periodic solutions: the period increases (decreases) if $T_{2}>0\left(T_{2}<0\right)$.

\section{Application to Control of Chaos}

In this section, we apply the results in the previous sections to system (2) for the purpose of control of chaos. From Section 2, we know that, under certain conditions, a family of periodic solutions bifurcate from the steady states of system (2) at some critical values of $\tau$ and the stability of the steady state maybe changes along with increasing of $\tau$. If the bifurcating periodic solution is orbitally asymptotically stable or some steady state becomes locally stable, then chaos may vanish. Let $a=10, b=33$, and $c=40$, and system (2) will become the following delayed feedback control form:

$$
\begin{aligned}
& \dot{x}(t)=40(y(t)-x(t)), \\
& \dot{y}(t)=-40 x(t)-33 y(t) z(t)+k[y(t)-y(t-\tau)], \\
& \dot{z}(t)=-10+y^{2}(t),
\end{aligned}
$$

which has the equilibria $E_{1}(\sqrt{10}, \sqrt{10},-40 / 33), E_{2}(-\sqrt{10}$, $-\sqrt{10},-40 / 33)$, and the linearized system of system (68) at $E_{1,2}$ is

$$
\begin{gathered}
\dot{u}(t)=u(t)\left(\begin{array}{ccc}
-40 & a & 0 \\
-40 & k+40 & -33 \sqrt{10} \\
0 & 2 \sqrt{10} & 0
\end{array}\right) \\
+u(t-\tau)\left(\begin{array}{ccc}
0 & 0 & 0 \\
0 & -k & 0 \\
0 & 0 & 0
\end{array}\right)
\end{gathered}
$$

where $u(t)=(x(t), y(t), z(t))^{T}$.

The characteristic equation of (69) is given by

$$
\begin{gathered}
\lambda^{3}-k \lambda^{2}+(660-40 k) \lambda+26400 \\
+\left(k \lambda^{2}+40 k \lambda\right) e^{-\lambda \tau}=0 .
\end{gathered}
$$

When $k=0, \tau=0$ (70) becomes

$$
\lambda^{3}+25 \lambda^{2}+1660 \lambda+26400-25 \lambda^{2}-1000 \lambda=0 .
$$

From Routh-Hurwitz criterion, it is easy to know that the equilibrium $E_{1,2}$ is unstable, and, using Matlab 7.8.0, we can get chaotic phenomena (see Figure 1).

In the following, we discuss the condition under which Hopf bifurcation occurs at the equilibria $E_{1,2}$; supposing $i \omega$ is a root of (71), we get

$$
\begin{aligned}
\omega^{6}- & 2(660-40 k) \omega^{4} \\
& +\left[(660-40 k)^{2}-226400(-k)-(40 k)^{2}\right] \omega^{2} \\
& +696960000=0 .
\end{aligned}
$$




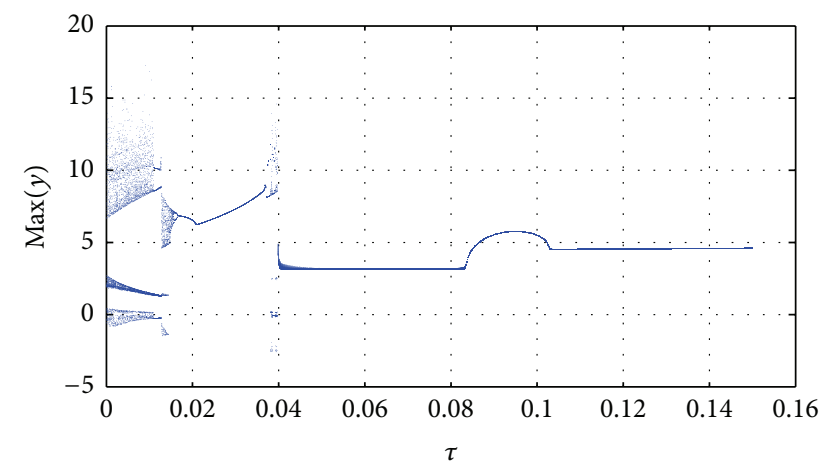

FIGURE 2: Bifurcation diagram of system (2) with $\tau \in(0.0001,0.15)$.

Let $z=\omega^{2}$; (72) becomes

$$
\begin{aligned}
z^{3}-2 & (660-40 k) z^{2} \\
+ & {\left[(660-40 k)^{2}-226400(-k)-(40 k)^{2}\right] z } \\
+ & 696960000=0 .
\end{aligned}
$$

Let $p=-2(660-40 k), q=\left[(660-40 k)^{2}-226400(-k)-\right.$ $\left.(40 k)^{2}\right]$, and $r=a_{0}^{2}, K=-25$; then $\Delta=p^{2}-3 q=[-2(660-$ $40 k)]^{2}-3\left[(660-40 k)^{2}-226400(-k)-(40 k)^{2}\right]=22735600>0$.

Equation (73) has two real roots:

$$
\begin{aligned}
z_{1} & \approx 3107.7, \\
\omega_{1} & \approx 55.7467, \\
\tau_{1}^{j} & \approx 0.0292+\frac{2 j \pi}{\omega_{1}}, \\
h^{\prime}\left(z_{1}\right) & \approx 8.7739 e^{+006}>0, \\
z_{2} & \approx 591.5, \\
\omega_{2} & \approx 24.3208, \\
\tau_{2}^{j} & \approx 0.0397+\frac{2 j \pi}{\omega_{2}}, \\
h^{\prime}\left(z_{2}\right) & \approx-2.4423 e^{+006}<0 .
\end{aligned}
$$

From Lemma 3 we have

$$
\begin{aligned}
& \frac{\operatorname{Re} \lambda\left(\tau_{1}^{j}\right)}{d \tau}>0, \\
& \frac{\operatorname{Re} \lambda\left(\tau_{2}^{j}\right)}{d \tau}<0 .
\end{aligned}
$$

In addition, notice that $\tau_{1}^{0}=0.0292<\tau_{2}^{0}=0.0397<\tau_{1}^{1}=$ $0.083<\tau_{2}^{1}=0.166 \ldots$..

By Theorem 4 , the equilibria points $E_{1,2}$ are unstable; when $\tau \in\left[0, \tau_{2}^{0}\right.$ ) (see Figures 2 and 4 ), the system varies from the chaos to the inverse double period bifurcation, the unstable bifurcating period solution, and double period

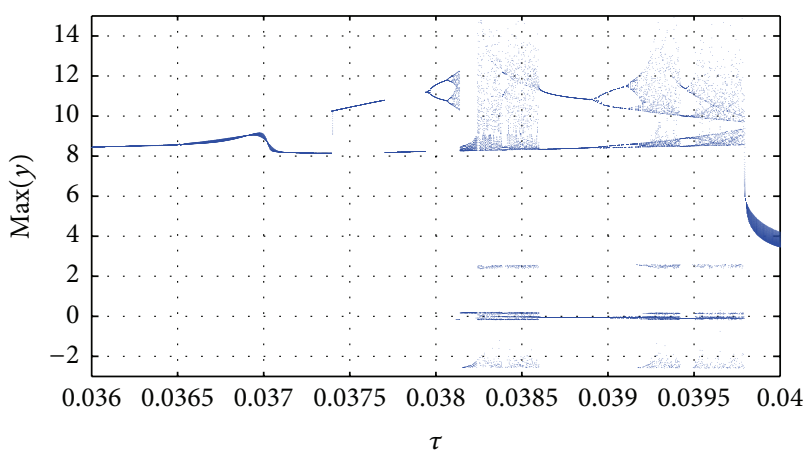

FIgURE 3: Local amplification of Figure 2 with $\tau \in(0.036,0.04)$.
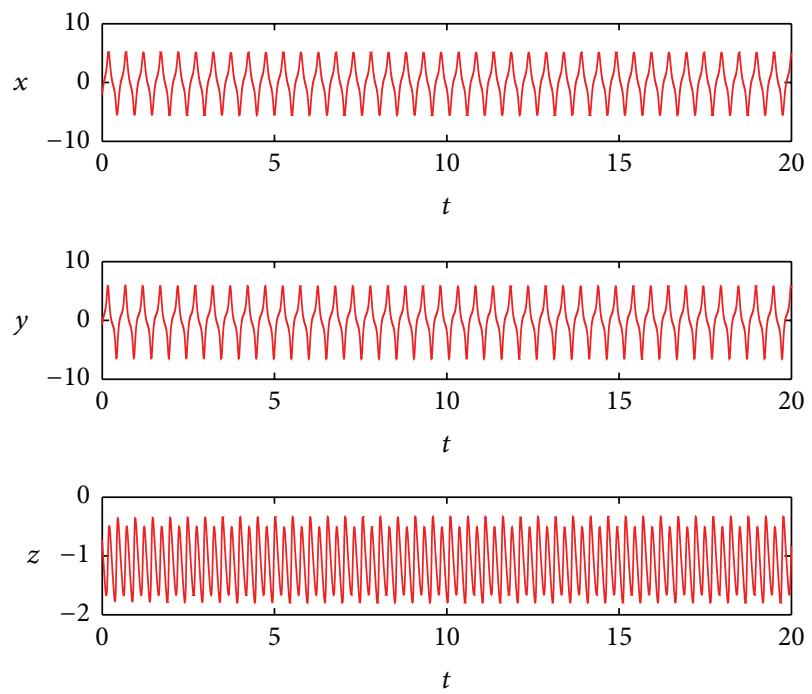

(a) The trajectories

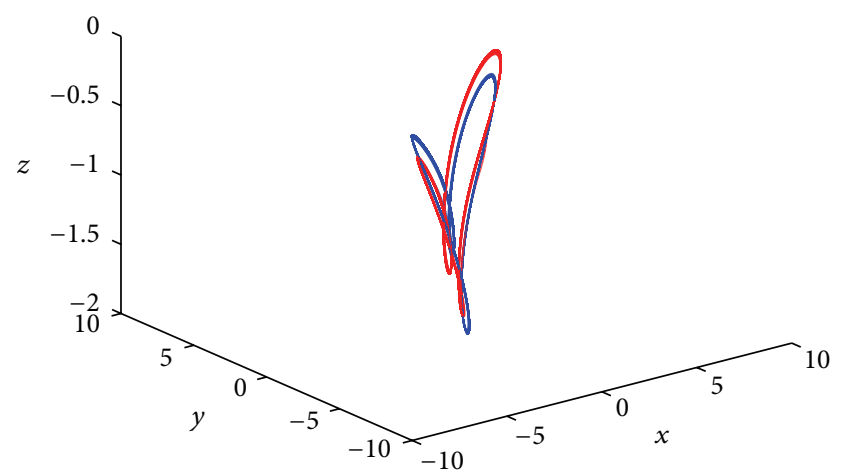

(b) Phase graph

FIGURE 4: The trajectories and phase graphs of system (2) with $\tau=0.02<0.0397, k=-25$ at initial values of phase graphs: $E_{1}(3.1,3.1,-1.1)(\mathrm{red}), E_{2}(-3.1,-3.1,-1.1)$ (blue).

bifurcation and the chaos occurs from the equilibria $E_{1,2}$ (see Figures 2 and 3). When $\tau$ pass through $\tau_{1}^{0}=0.0397$, Hopf bifurcation occurs. When $\tau \in\left[\tau_{2}^{0}, \tau_{1}^{1}\right)$, the equilibria $E_{1,2}$ are asymptotically stable (see Figure 5). When $\tau>$ 0.083 , the equilibria $E_{1,2}$ lose their stability and the stable bifurcating period solutions occur (see Figure 6). By the 


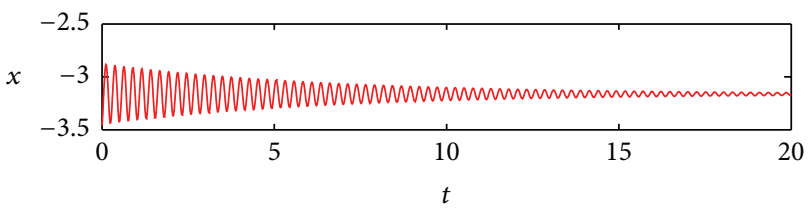

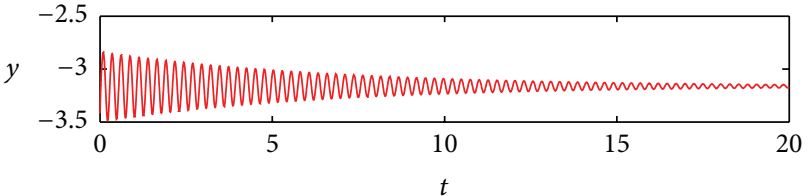

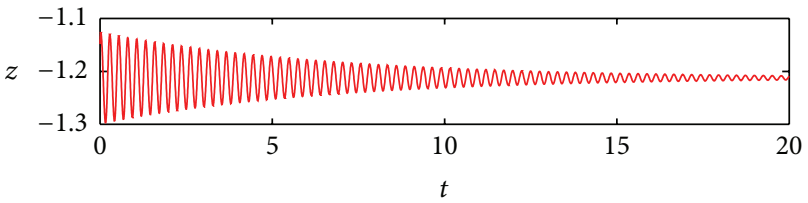

(a) The trajectories

FIgURE 5: The trajectories and phase graphs of system with $\tau=0.0405>0.0397, k=-25$ at initial values of phase graphs: $E_{1}(3.1,3.1,-1.1)$ (red), $E_{2}(-3.1,-3.1,-1.1)$ (blue).
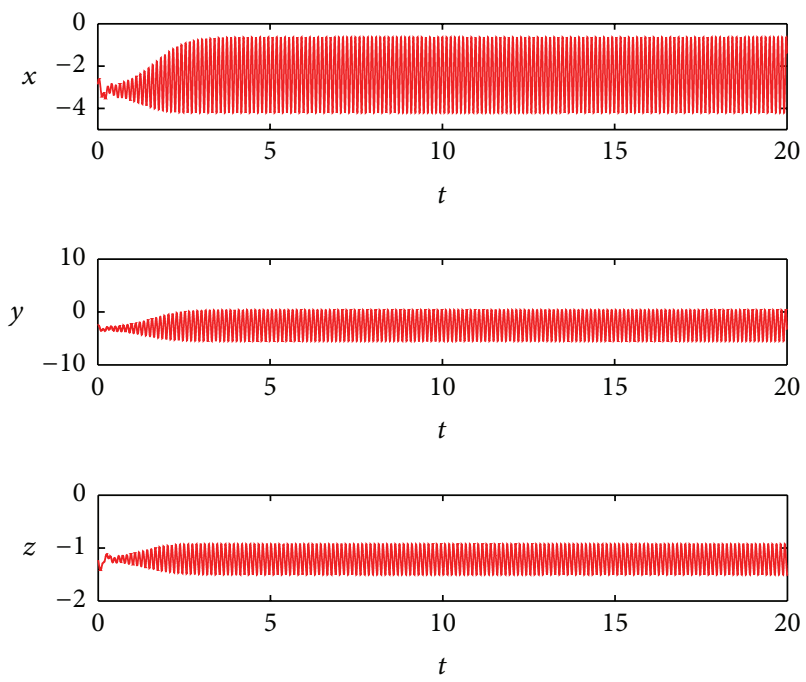

(a) The trajectories

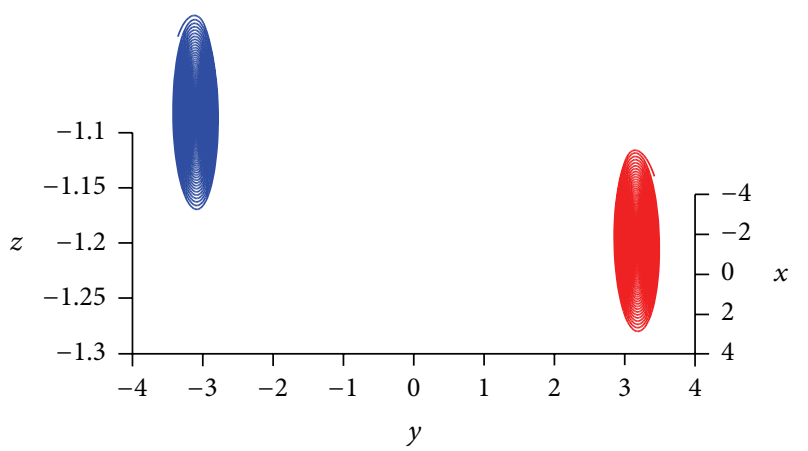

(b) Phase graph (b) Phase graph

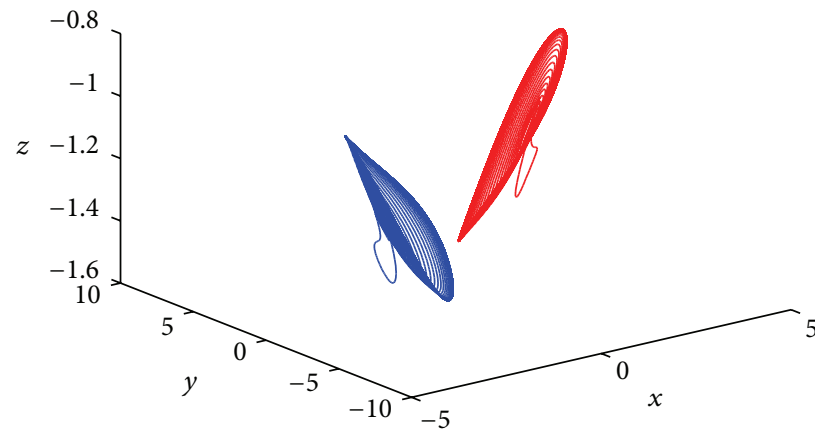

FiguRE 6: The trajectories and phase graphs of system (2) with $\tau=0.09, k=-25$ at initial values of phase graphs: $E_{1}(3.1,3.1,-1.1)($ red), $E_{2}(-3.1,-3.1,-1.1)$ (blue).

theory of Hale [11], from formula (62), we also determine the direction of Hopf bifurcation and the other properties of bifurcating periodic solutions. From the formulae in Section 4 we evaluate $C_{1}(0), \mu_{2}, \beta_{2}$, and $T_{2}$; it follows that

$$
\begin{aligned}
C_{1}(0) & \approx-0.0102-0.0073 i, \\
\mu_{2} & \approx-3.0600 e^{-005}<0, \\
\beta_{2} & \approx-0.0204<0, \\
T_{2} & \approx-0.0076<0 .
\end{aligned}
$$

Since $\mu_{2}<0, \beta_{2}<0$, and $T_{2}<0$, the Hopf bifurcation is subcritical; these bifurcating period solutions from $E_{1,2}$ are stable and the period of the bifurcating periodic solutions decreases.

\section{Conclusions}

In this paper, our goal is to control a chaotic system to be of stable state by applying the time-delayed feedback control method. It is shown that time delays play an important role in controlling the chaotic behaviors of system (1). The sufficient conditions are obtained to ensure local stability of the equilibria $E_{1}, E_{2}$ and the existence of local Hopf bifurcation. In virtue of functional differential equations and Hassard method, the nature of the Hopf bifurcation of the time delay chaotic system is determined by the designed 
controller. Numerical simulation results demonstrate that the new feedback controller using the time delay is a specially effective method to control chaos behavior. Through further investigation, we expect to decide on which bifurcation arises in the chaos system by proper setting on the feedback parameters for building programs to suppress chaos.

\section{Conflict of Interests}

The authors declare that there is no conflict of interests regarding the publication of this paper.

\section{Acknowledgments}

The authors would like to thank the anonymous referee for the very helpful suggestions and comments which led to the improvement of the original paper. And this work is supported by the Education Department of Henan Province (Grant no. 15A110046) and the Research Innovation Project of Zhoukou Normal University (Grant nos. ZKNUA201410 and ZKNU2014126).

\section{References}

[1] M. Adimy, F. Crauste, and S. Ruan, "Stability and Hopf bifurcation in a mathematical model of pluripotent stem cell dynamics," Nonlinear Analysis: Real World Applications, vol. 6, no. 4, pp. 651-670, 2005.

[2] H. N. Agiza, "Controlling chaos for the dynamical system of coupled dynamos," Chaos, Solitons and Fractals, vol. 13, no. 2, pp. 341-352, 2002.

[3] F. M. Atay, "Delayed feedback control near Hopf bifurcation," Discrete and Continuous Dynamical Systems, vol. 1, pp. 197-205, 2008.

[4] E.-W. Bai and K. E. Lonngren, "Sequential synchronization of two Lorenz systems using active control," Chaos, solitons and fractals, vol. 11, no. 7, pp. 1041-1044, 2000.

[5] K. Balachandran, Y. Zhou, and J. Kokila, "Relative controllability of fractional dynamical systems with delays in control," Communications in Nonlinear Science and Numerical Simulation, vol. 17, no. 9, pp. 3508-3520, 2012.

[6] G. Chen and X. Dong, From Chaos to Order C Perspectives, Methodologies, and Applications, World Scientific, Singapore, 1998.

[7] G. R. Chen and X. Dong, "On feedback control of chaotic continuous-time systems," IEEE Transactions on Circuits and Systems. I. Fundamental Theory and Applications, vol. 40, no. 9, pp. 591-601, 1993.

[8] M. M. El-Dessoky, M. T. Yassen, and E. S. Aly, "Bifurcation analysis and chaos control in Shimizu-Morioka chaotic system with delayed feedback," Applied Mathematics and Computation, vol. 243, pp. 283-297, 2014.

[9] Z. Elhadj and J. C. Sprott, "Non-existence of Shilnikov chaos in continuous-time systems," Applied Mathematics and Mechanics, vol. 33, no. 3, pp. 371-374, 2012.

[10] Q. Han, "On designing time-varying delay feedback controller for master-slave synchronization of Lure systems," IEEE Transactions on Circuits and Systems-I: Fundamental Theory and Applications, vol. 54, no. 7, pp. 1573-1583, 2007.
[11] J. K. Hale, Theory of Functional Differential Equation, Springer, Berlin, Germany, 1977.

[12] Y. Kuang, Delay Differential Equations: With Applications in Population Dynamics, Academic Press, San Diego, Calif, USA, 1993.

[13] K. K. Pyragas, "Continuous control of chaos by self-controlling feedback," Physics Letters A, vol. 170, no. 6, pp. 421-428, 1992.

[14] J. A. K. Suykens, P. F. Curran, and L. O. Chua, "Master-slave synchronization using dynamic output feedback," International Journal of Bifurcation and Chaos, vol. 7, no. 3, pp. 671-679, 1997.

[15] L. Zhou and F. Chen, "Hopf bifurcation and Si'lnikov chaos of Genesio system," Chaos, Solitons \& Fractals, vol. 40, no. 3, pp. 1413-1422, 2009.

[16] H. Zhao and L. Wang, "Hopf bifurcation in Cohen-Grossberg neural network with distributed delays," Nonlinear Analysis, vol. 8, no. 1, pp. 73-89, 2007.

[17] H. T. Zhao, Y. P. Lin, and Y. X. Dai, "Bifurcation analysis and control of chaos for a hybrid ratio-dependent three species food chain," Applied Mathematics and Computation, vol. 218, no. 5, pp. 1533-1546, 2011.

[18] M. T. Yassen, "Chaos control of Chen chaotic dynamical system," Chaos, Solitons and Fractals, vol. 15, no. 2, pp. 271-283, 2003.

[19] X. Wang and G. Chen, "A chaotic system with only one stable equilibrium," Communications in Nonlinear Science and Numerical Simulation, vol. 17, no. 3, pp. 1264-1272, 2012.

[20] X. Wang and G. Chen, "Coexistence of point, periodic and sreange attractors," International Journal of Bifurcation and Chaos, vol. 23, no. 5, Article ID 1350093, 5 pages, 2013.

[21] X. Wang and G. Chen, "A chaotic system with only one stable equilibrium," Nonlinear Dynamics, vol. 71, pp. 429-436, 2013.

[22] S. Jafari and J. C. Sprott, "Simple chaotic flows with a line equilibrium," Chaos, Solitons \& Fractals, vol. 57, pp. 79-84, 2013.

[23] B. Hassard, N. Kazarinoff, and Y. Wan, Theorem and Application of Hopf Bifurcation, Cambridge University Press, Cambridge, UK, 1981.

[24] J. Lü and G. Chen, "A new chaotic attractor coined," International Journal of Bifurcation and Chaos, vol. 12, no. 3, pp. 659661, 2002.

[25] W. Zhou, Y. Xu, H. Lu, and L. Pan, "On dynamics analysis of a new chaotic attractor," Physics Letters A, vol. 372, no. 36, pp. 5773-5777, 2008.

[26] S. Ruan and J. Wei, "On the zeros of a third degree exponential polynomial with application to delayed model for the control of testosterone secretin," IMA Journal of Mathemathics Applied in Medicine and Biology, vol. 18, no. 1, pp. 41-52, 2001. 


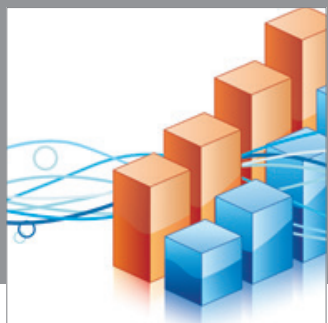

Advances in

Operations Research

mansans

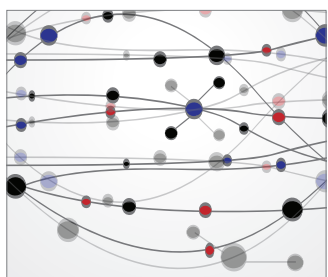

The Scientific World Journal
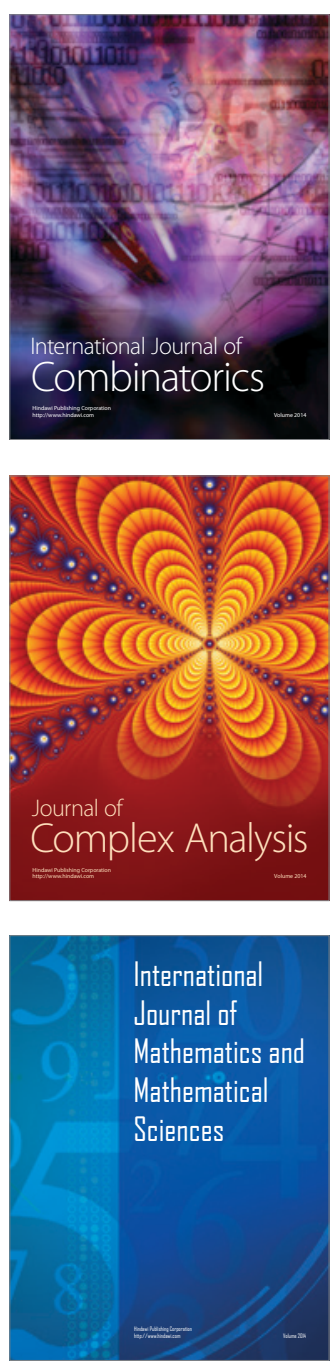
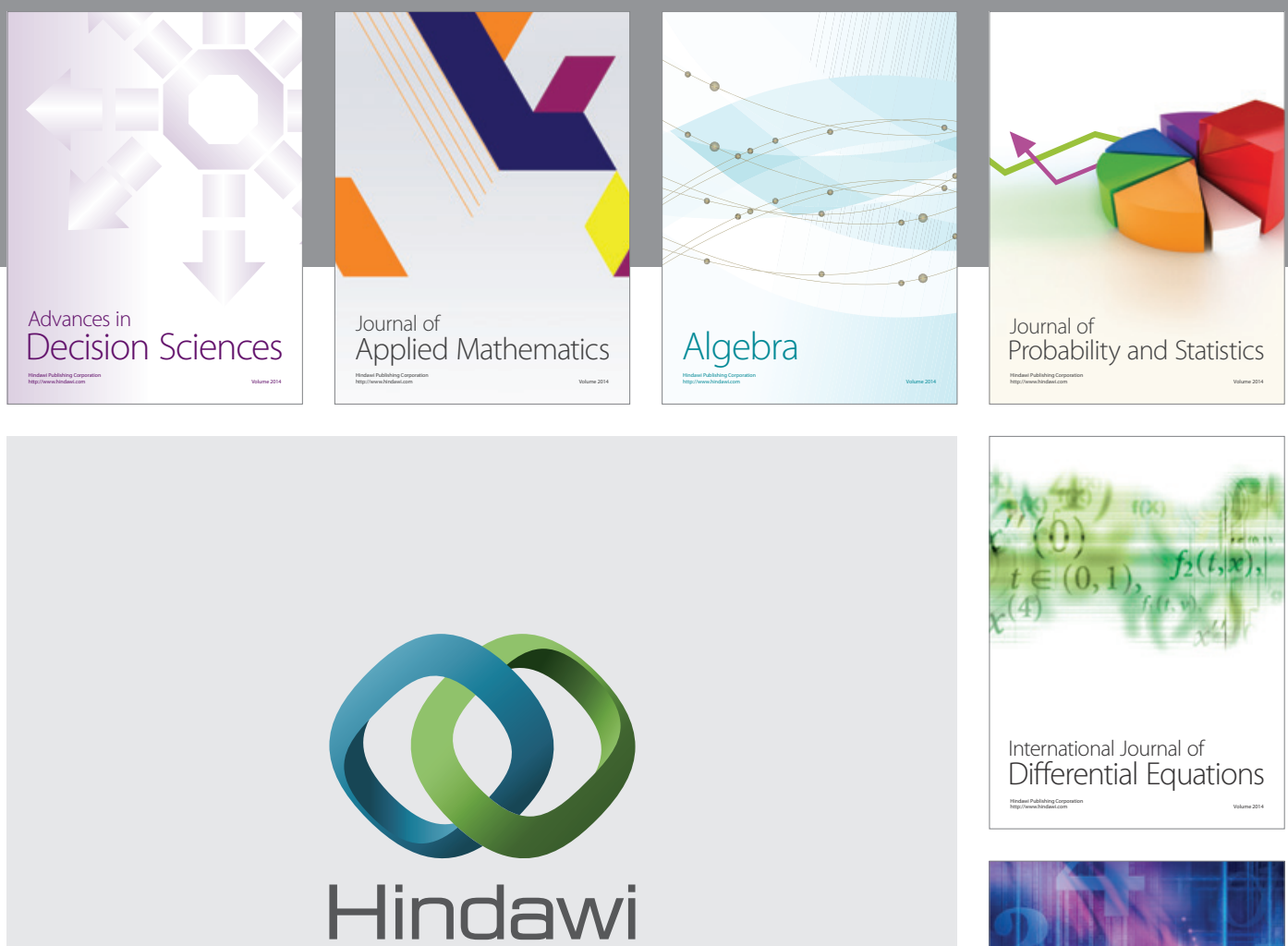

Submit your manuscripts at http://www.hindawi.com
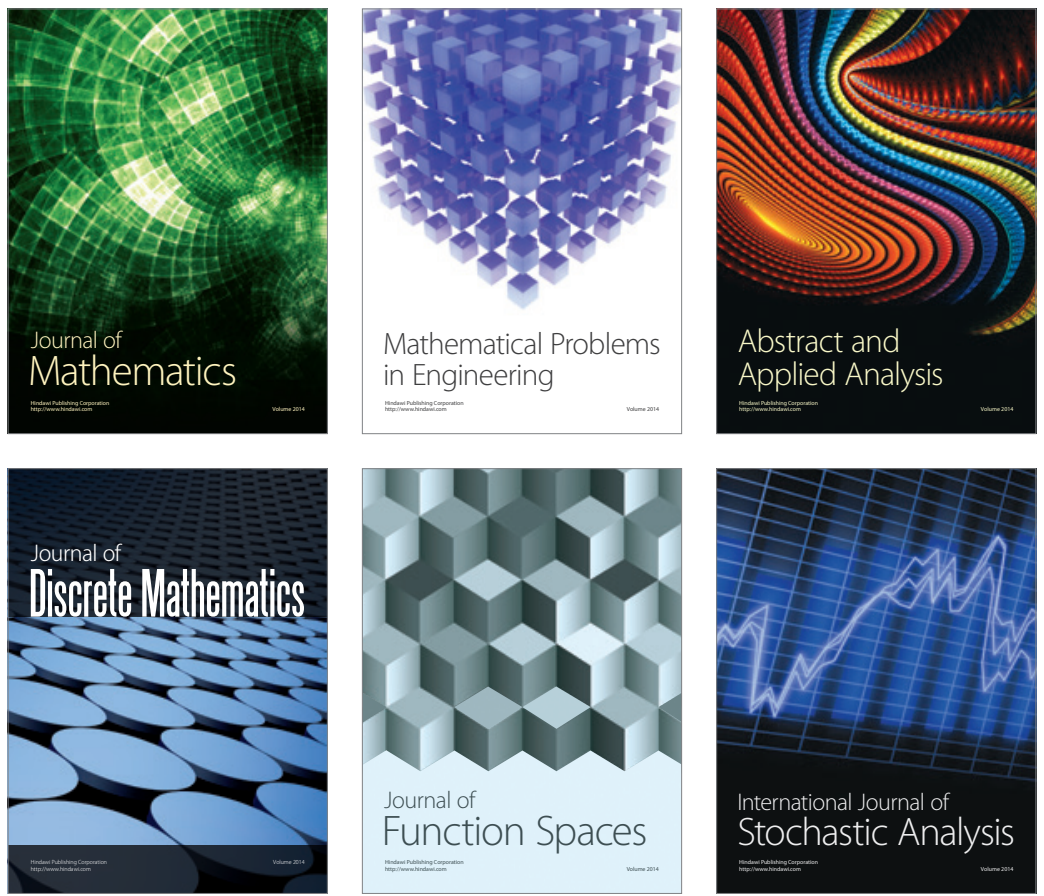

Journal of

Function Spaces

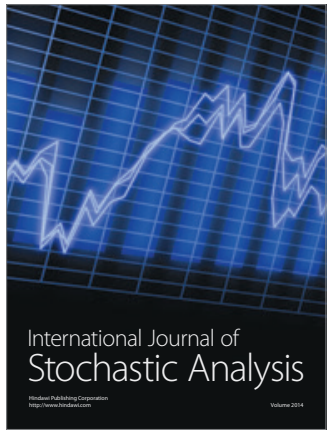

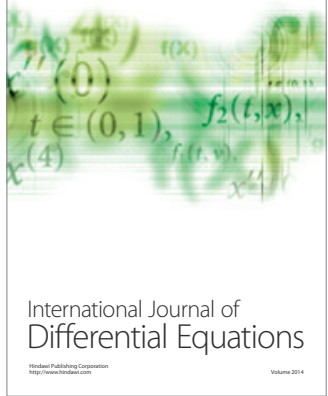
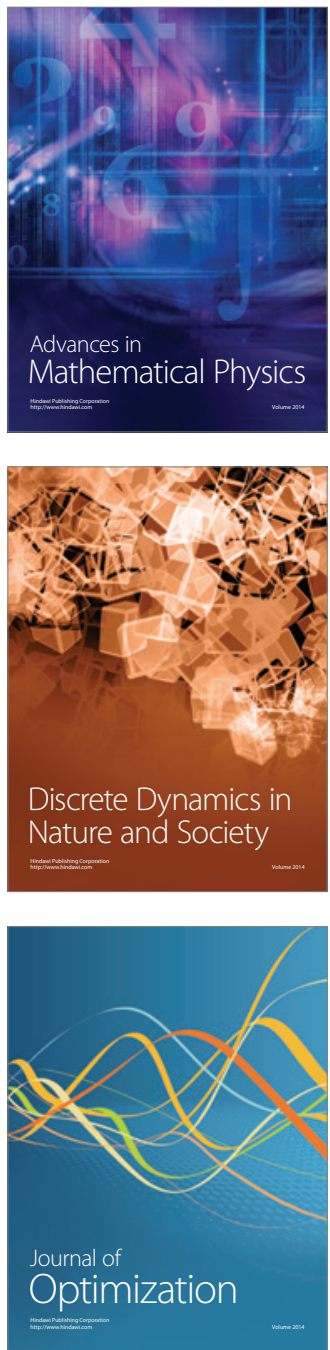